\title{
Calsiara Tablets: A Clinical Study on Calcium Citrate malate for Bone Health
}

Dr. M. Sampoorna, S. V. Bhavani, Asst. Prof. K. Jayasurya \& Asst. Prof. Badugu Kranthi kumar

Besiara Pharmaceuticals, 4-77/2, Badangpet, Hyderabad, Telangana, 500058, India.

www.besiara.com

DOI: http://doi.org/10.38177/ajast.2020.4411

Copyright: $\odot 2020$ M. Sampoorna et al. This is an open access article distributed under the terms of the Creative Commons Attribution License, which permits unrestricted use, distribution, and reproduction in any medium, provided the original author and source are credited.

\section{ABSTRACT}

Calcium citrate malate is most easily absorbed by the body. Calcium is essential for many diverse processes in the body, including bone formation, muscle contraction, and enzyme and hormone functioning. Calcium is very essential in muscle contraction, oocyte activation, building strong bones and teeth, blood clotting, nerve impulse, transmission, regulating heart beat and fluid balance within cells. Calcium supplementation in pregnancy has the potential to reduce adverse gestational outcomes, in particular by decreasing the risk of developing hypertensive disorders during pregnancy, which are associated with a significant number of maternal deaths and considerable risk of preterm birth, the leading cause of early. Calcium citrate maleate and other nutrients are essential for cell growth and the production and functioning of DNA - the body's genetic blueprint. Based on clinical evidences Calsiara $^{\text {TM }}$ is essential for Pregnancy Mother and Fetal development. Along with to improve the mineral density of bone.

Keywords: Calcium citrate malate, vitamin D3, Human milk oligosaccharides (HMOs), Magnesium, premature labor, zinc, Copper, Pregnancy, Fetal development, New born.

\section{Aim and Objective}

Aim

The aim of the study was to determine the use of Calcium citrate malate and other components like Zinc, Copper, Magnesium and Vit D3 in Pregnancy and fetal development.

\section{Objective}

To evaluate the usefulness of supplementations like vitamin D3, fructooligosaccharides, magnesium, zinc, manganese, copper, Calcium citrate malate for fetal development in the Pregnancy.

\section{Introduction}

\section{Calcium Citrate Malate}

Calcium citrate malate may be used to treat conditions caused by low calcium levels such as bone loss (osteoporosis), weak bones (osteomalacia/rickets), decreased activity of the parathyroid gland. Your doctor may recommend a calcium citrate supplement if you are having trouble getting enough calcium or you are at risk for high blood pressure in pregnancy. Calcium citrate malate is most easily absorbed by the body. Calcium citrate malate may also be used in certain patients to make sure they are getting enough calcium (including women who are pregnant, nursing, or postmenopausal, people taking certain medications such as phenytoin, phenobarbital, or prednisone). Calcium plays a very important role in the body. It is necessary for normal functioning of nerves, cells, muscle, and bone. If there is not enough calcium in the blood, then the body will take calcium from bones, thereby weakening bones. Dairy products are considered some of the best sources of calcium, but they aren't your only 
choice. If you're lactose intolerant, don't eat animal products, or you just don't like milk, you can still get enough calcium without dairy.

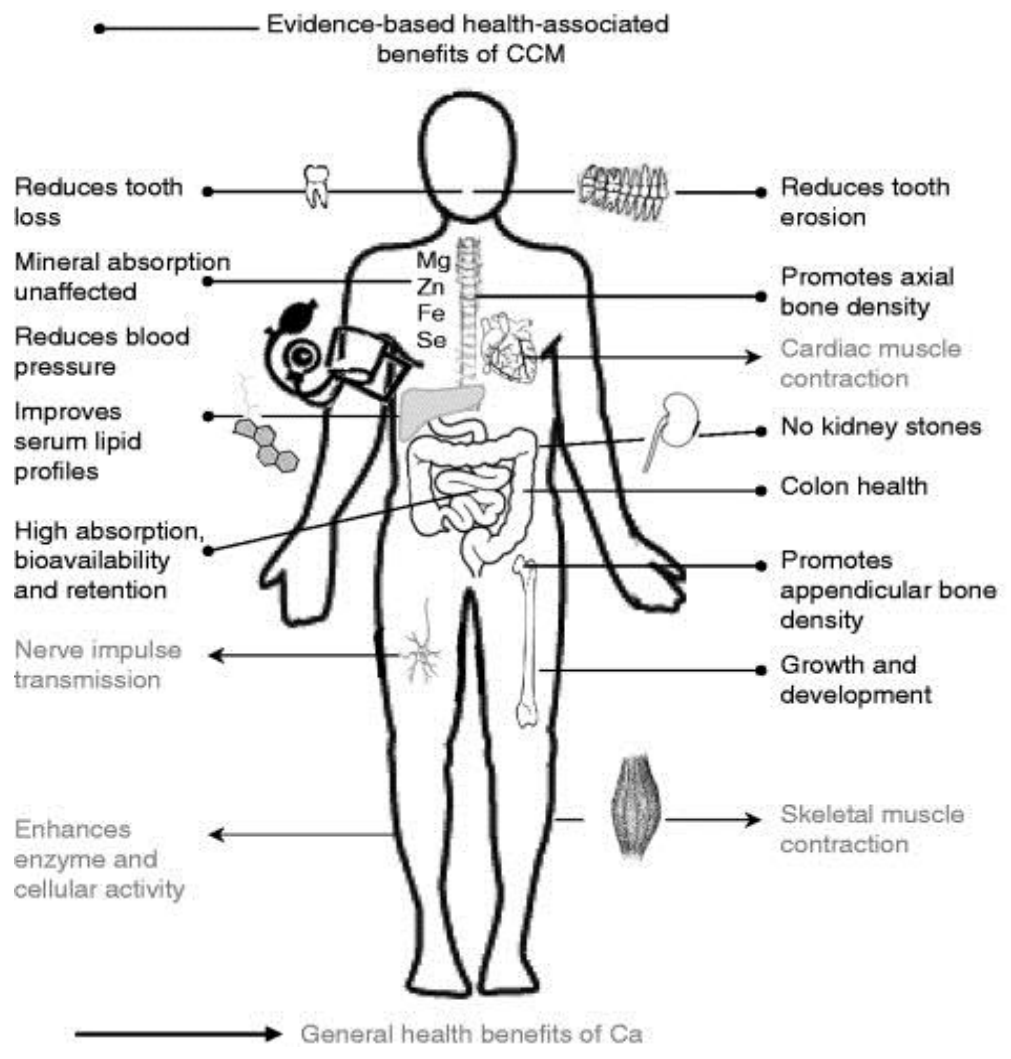

Calcium is essential for many diverse processes in the body, including bone formation, muscle contraction, and enzyme and hormone functioning. Inadequate calcium consumption by pregnant women can lead to adverse effects in both the mother and the fetus and produce:

\section{$>$ Osteopenia}

$>$ Tremor

$>$ Paraesthesia

$>$ Muscle cramping

$>$ Tetanus

$>$ Delayed fetal growth

$>$ Low birth weight

$>$ Poor fetal mineralization

Calcium supplementation has shown to produce a beneficial effect in reducing the risk of pregnancy-induced hypertension. Calcium in supplements may come in the form of carbonate, citrate, lactate or gluconate, and in general has good bioavailability. Supplements are inexpensive and readily accessible. Calcium supplementation in pregnancy has the potential to reduce adverse gestational outcomes, in particular by decreasing the risk of 
Asian Journal of Applied Science and Technology

Volume 4, Issue 4, Pages 130-138, October-December 2020

developing hypertensive disorders during pregnancy, which are associated with a significant number of maternal deaths and considerable risk of preterm birth, the leading cause of early.

Calcium citrate malate is one of the key minerals you need during pregnancy along with other vitamins and minerals, your body provides it to your baby to aid the development of vital structures like the skeleton. Calcium is a mineral that is found naturally in foods. Calcium is necessary for many normal functions of the body, especially bone formation and maintenance. Calcium citrate is used to prevent and to treat calcium deficiencies.

\section{Daily Requirements}

Teen moms require a little more. They need enough to maintain their bones and the stores of calcium in their own bodies while supporting the growth of their baby. Therefore, experts recommend that pregnant teens aged 18 and under get at least $1,300 \mathrm{mg}$ of calcium each day.

\section{For Your Baby}

Your developing baby needs calcium to form bones and teeth. They're building an entire skeleton, after all. Calcium is also an important nutrient for your baby's heart, muscles, nerves, and hormones.

\section{For You}

During pregnancy, you give your baby all the calcium they need, so when you consume the recommended amount of calcium every day you are taking care of your baby and yourself. If you don't get enough, you could run into some complications. Regardless of whether or not you take in enough, your body will still give calcium to your baby. So, if you are not replacing what you're giving away, you could end up with weakened bones and a greater risk of osteoporosis later in life.

\section{Complications}

Not consuming enough calcium can lead to:

High blood pressure during pregnancy

$>$ Premature birth

$>$ Low birth weight

$>$ Numbness and tingling in the fingers

$>$ Slow growth of the baby

$>$ The baby not getting enough calcium in the bones

$>$ Heart problems

$>$ Muscle and leg cramps

$>$ A poor appetite

$>$ In rare cases, increased risk of bone fractures. 


\section{Sources}

$>$ Fortified milk or other fortified dairy products will fulfill your daily calcium requirement

$>$ A glass of fortified orange juice

$>$ Other foods such as greens, nuts, and beans

$>$ Cheese

$>$ Yogurt

$>$ Collard green

$>$ Broccoli

$>$ Bok choy

$>$ Soybeans

$>$ Baked beans

$>$ Almonds

$>$ Salmon

$>$ Dark leafy greens

Make sure the dairy products you eat are pasteurized and talk to your doctor about the type of milk and dairy products that are best. Low-fat and non-fat milk contains all the calcium and nutrients of whole milk without the extra fat and calories. However, your doctor will advise you on the best choice based on whether you are underweight, within the recommended weight range, or overweight. Your doctor may recommend a calcium citrate supplement if you are having trouble getting enough calcium or you are at risk for high blood pressure in pregnancy.

\section{Vitamin D3}

Vitamin D3 is known to play an important role in bone metabolism through regulation of calcium and phosphate equilibrium. Insufficient vitamin D3 linked to miscarriage among women with prior pregnancy loss. NIH study suggests the importance of preconception vitamin D in maintaining pregnancy. Vitamin D supplementation during pregnancy improves maternal vitamin D status and may reduce the risk of pre-eclampsia, low birthweight and preterm birth. A deficiency in vitamin D can result from inadequate exposure to sunlight, inefficient production in the skin, not enough vitamin D in your diet, and health conditions that can affect Pregnancy including, gastrointestinal disorders, renal diseases, and liver diseases.

To get the vitamin D you need each day, you can:

Spend a little time in the sun. Sunlight converts a chemical in your skin to vitamin D. 
Asian Journal of Applied Science and Technology

Volume 4, Issue 4, Pages 130-138, October-December 2020

$\checkmark$ Consume fortified products. Many calcium-rich foods are also fortified with vitamin D such as milk, orange juice, cereal, yogurt, and eggs.

$\checkmark$ Take your prenatal vitamin. Most prenatal vitamins contain vitamin D. But, be sure to read

\section{Fructooligosaccharides}

Fructooligosaccharides (FOS) are oligosaccharides that occur naturally in plants such as onion, chicory, garlic, asparagus, banana, artichoke, among many others. They are composed of linear chains of fructose units, linked by beta (2-1) bonds. Fructooligosaccharides (FOS), which are low-calorie, non-digestible carbohydrates that can improve food taste and texture while aiding immunity, bone health and the growth and balance of important bacteria in the digestive track. Human milk oligosaccharides (HMOs) circulate during early pregnancy in the maternal blood. We show that mothers produce HMOs starting as early as 10 weeks of gestation and thus, months before the baby is born. This suggests that babies might already benefit from HMOs before they take their first sip of mother's milk. Whether HMOs protect the unborn indirectly, e.g. by protecting the mother from infections or facilitating immune and metabolic adaptations during pregnancy, or whether HMOs have direct benefits on the unborn, remains to be elucidated. Our studies show that HMOs profiles change over the course of pregnancy in concentration and composition. Human milk oligosaccharides (HMOs) are bioactive glycans linked with health benefits to both the breast-fed infant and lactating mother. HMOs are present before lactation, already during pregnancy, and are influenced by maternal body composition. HMO concentration increased with gestational age. Human milk oligosaccharides (HMOs) are highly abundant, bioactive glycans in human milk that offer a variety of potential benefits to the breast-fed infant (3). The best studied HMO effects are their topical effects in the infant gut, such as prebiotic effects on beneficial bacteria and antiadhesive/antimicrobial functions against pathogens.

\section{Magnesium}

Magnesium $(\mathrm{Mg})$ is an essential mineral required to regulate body temperature, nucleic acid, and protein synthesis with an important role in maintaining nerve and muscle cell electrical potentials. It may reduce fetal growth restriction and preeclampsia as well as increase birth weight. Deficiency in this mineral during pregnancy may increase the risk of chronic hypertension and premature labor. Some studies suggest that supplementing with magnesium may reduce the risk of complications like fetal growth restriction and preterm birth. Magnesium and calcium work in combination: Magnesium relaxes muscles, while calcium stimulates muscles to contract. Research suggests that getting adequate magnesium during pregnancy can help prevent the uterus from contracting prematurely. Magnesium also helps build strong teeth and bones in your baby. Low magnesium is associated with increased risk of miscarriage. Low magnesium may also be associated with birth defects. Multivitamins: lowered the risk of miscarriage by $57 \%$ in one study.

\section{Zinc}

Your baby needs zinc for cell growth and for the production and functioning of DNA - the body's genetic blueprint. Getting enough zinc is especially important during pregnancy because there's so much rapid cell growth. This 
essential mineral also helps support your immune system, maintain your senses of taste and smell, and heal wounds. Zinc deficiency to low birth weight and other problems during pregnancy, labor, and delivery.

\section{Manganese}

Manganese is a mineral that helps form bone and cartilage. It also helps protect cells from damage and activates the enzymes that play a role in the metabolism of carbohydrates, amino acids, and cholesterol.

\section{Copper}

Copper, a trace mineral found in all plant and animal tissues, is essential for forming red blood cells. This is especially important during pregnancy, when your blood supply doubles. Copper helps form your baby's heart, blood vessels, and skeletal and nervous systems. Drinking 2 to 3 glasses of water that has been stored in a copper vessel is another easy way to supply your body with enough copper. According to Ayurveda, drinking copper-enriched water first thing in the morning on an empty stomach helps balance all three doshas (Kapha, Vata and Pitta).

\section{Literature Review}

Ambrish Mithal, et al, (2014): Vitamin D deficiency and insufficiency are common across the globe. Large epidemiological studies reveal the high prevalence of vitamin D in women, including antenatal and lactating mothers. Vitamin D requirements are probably greater in pregnancy, as evidenced by physiologically higher 1,25-dehydroxy vitamin D levels seen in the second and third trimesters. While 1,25(OH) 2D levels do not correlate directly with 25 hydroxy vitamin D concentrations, the physiological rise in the active metabolite, the enhanced intestinal calcium absorption, and enhanced fetal requirement of calcium (250 $\mathrm{mg} /$ day in the third trimester) all point to the importance of vitamin D biology in pregnancy. The musculoskeletal manifestations of vitamin D deficiency are well known: Rickets and osteomalacia have been linked with the condition for nearly a century now. Myriad metabolic, nonskeletal associations of vitamin D deficiency are now being unraveled as well. Various authors report links between low vitamin D levels and various elements of the metabolic syndrome. Yet others describe the immunomodulatory, anabolic, anti-infective and anti-tumoral potential of vitamin D. Maternal secondary hyperparathyroidism and osteomalacia, neonatal hypocalcemia and tetany, delayed ossification of the cranial vertex, enlarged size of cranial, fontanelles, and impaired fetal bone ossification has been reported by various authors, and reviewed in detail by others. The relationship between low vitamin D and adverse maternal outcomes such as pregnancy induced hypertension high blood pressure in diabetic pregnancy gestational diabetes mellitus recurrent pregnancy loss preterm delivery primary Caesarian section, and postpartum depression has been documented in recent years.

Sahu M, et al, (2009): Vitamin D deficiency is common in urban Indians despite living in the tropics and its public health consequences are enormous. However, $70 \%$ of India is rural, and data from rural subjects, who are expected to have good sun exposure, are scant. To determine the population prevalence of vitamin D deficiency in rural pregnant women and adolescent girls, compare serum 25-hydroxyvitamin D (25OHD) status in adolescent boys from the same families, and determine seasonal differences in serum 25OHD. A random selection of 121 
Asian Journal of Applied Science and Technology

Volume 4, Issue 4, Pages 130-138, October-December 2020

adolescent girls from a survey of a population of 8270 in a rural low socioeconomic community; 139 pregnant women in the second trimester; and a subset of 28 adolescent girls compared with 34 brothers. Serum 25OHD, serum alkaline phosphatase (AP), sun exposure, and dietary calcium intake. The age-adjusted community prevalence of vitamin D deficiency $(25 \mathrm{OHD}<50 \mathrm{nmol} / \mathrm{l})$ in adolescent girls was $88.6 \%$. Seventy-four per cent of pregnant women had vitamin D deficiency. Mean +/- SD 25OHD in girls and women in summer was 55.5 +/- 19.8 $\mathrm{nmol} / \mathrm{l}$ compared to $27.3+/-12.3 \mathrm{nmol} / \mathrm{l}$ in winter $(\mathrm{P}<0.001)$. Winter serum $25 \mathrm{OHD}$ in boys $(67.5+/-29.0 \mathrm{nmol} / \mathrm{l})$ was higher than that in their sisters $(31.3+/-13.5 \mathrm{nmol} / \mathrm{l}, \mathrm{P}<0.001)$. We report a high prevalence of vitamin $\mathrm{D}$ deficiency among pregnant women and adolescent girls from a rural Indian community. Boys are relatively protected. Seasonal variation in serum 25OHD is significant at latitude 26 degrees N.

Benjamin W, et al, (2012): Poor maternal zinc status has been associated with foetal loss, congenital malformations, intrauterine growth retardation, reduced birth weight, prolonged labor and preterm or post-term deliveries. A meta-analysis completed in 2007 showed that maternal zinc supplementation resulted in a small but significant reduction in preterm birth. The purposes of this analysis are to update that previous review and expand the scope of assessment to include maternal, infant and child health outcomes. Electronic searches were carried out to identify peer-reviewed, randomized controlled trials where daily zinc supplementation was given for at least one trimester of pregnancy. The co-authors applied the study selection criteria, assessed trial quality and abstracted data. A total of 20 independent intervention trials involving more than 11000 births were identified. The 20 trials took place across five continents between 1977 and 2008. Most studies assessed the zinc effect against a background of other micronutrient supplements, but five were placebo-controlled trials of zinc alone. The provided dose of supplemental zinc ranged from 5 to $50 \mathrm{mg} /$ day. Only the risk of preterm birth reached statistical significance (summary relative risk 0.86 [95\% confidence interval 0.75, 0.99]). There was no evidence that supplemental zinc affected any parameter of fetal growth (risk of low birth weight, birth weight, length at birth or head circumference at birth). Six of the 20 trials were graded as high quality. The evidence that maternal zinc supplementation lowers the risk of preterm birth was graded low; evidence for a positive effect on other fetal outcomes was graded as very low. The effect of zinc supplementation on preterm birth, if causal, might reflect a reduction in maternal infection, a primary cause of prematurity. While further study would be needed to explore this possibility in detail, the overall public health benefit of zinc supplementation in pregnancy appears limited.

\section{Conclusion}

We conclude that the best way to be sure that you're getting all the calcium and other nutrients that you need is to eat well. A balanced diet will keep you healthy during your pregnancy, provide your baby with what they need to grow and develop, give you strength and energy for childbirth, and encourage successful milk production once your child is born. Vitamin D3 supplementation during pregnancy improves maternal vitamin D3 status and may reduce the risk of pre-eclampsia, low birthweight and preterm birth. Human milk oligosaccharides (HMOs) are bioactive glycans linked with health benefits to both the breast-fed infant and lactating mother.

So Calsiara ${ }^{\text {TM }}$ is recommended for essential nutrient for pregnant mother and fetal development. Along with to improve the mineral density of bone. 


\section{References}

1. Hofmeyr G, Lawrie T, Atallah A, Duley L, Calcium supplementation during pregnancy for preventing hypertensive disorders and related problems, Cochrane Database Syst Rev, 2010, Vol no: 4, ISSN No: 8, Page no: CD001059.

2. Lonnerdal B. Calcium and iron absorption-mechanisms and public health relevance. Int J Vitam Nutr Res, 2010, Vol no: 80, ISSN No: 4-5, Page no: 293-299.

3. Merialdi M, Mathai M, Ngoc N, et al. World health organization systematic review of the literature and multinational nutritional survey of calcium intake during pregnancy, Fetal and Maternal Medicine Review, 2005, Vol no: 16, Page no: 97-121.

4. Palacios C, Pena-Rosas J. Calcium supplementation during pregnancy for preventing hypertensive disorders and related problems: RHL commentary. Geneva, The World Health Organization Reproductive Health Library, 2010, Vol no: 7, Page no: 110-119.

5. Ambrish Mithal, Sanjay Kalra, Vitamin D supplementation in pregnancy, Indian J Endocrinol Metab, 2014 Sep-Oct, Vol no: 18, ISSN No: 5, Page no: 593-596.

6. Kalra S, Malik S, John M, Gestational diabetes mellitus: A window of opportunity, Indian J Endocrinol Metab, 2011, Vol no: 15, Page no: 149-51.

7. Sahu M, Bhatia V, Aggarwal A, Rawat V, Saxena P, Pandey A, et al. Vitamin D deficiency in rural girls and pregnant women despite abundant sunshine in northern India, Clin Endocrinol (Oxf) 2009, Vol no: 70, Page no: $680-4$.

8. Lais Vales Mennitti, Lila Missae Oyama, Juliana Lopez de Oliveira, Ana Claudia Losinskas Hachul, Aline Boveto Santamarina, Aline Alves de Santana, Oligofructose supplementation during pregnancy and lactation impairs offspring development and alters the intestinal properties of 21-d-old pups, Lipids Health Dis, 2014, Vol no: 13, Page no: 26.

9. Sare Bakouei, Fatemeh Reisian, Minoor Lamyian, Ebrahim Haji Zadeh, Hadi Zamanian, Zahra Taheri Kharameh, High Intake of Manganese During Second Trimester, Increases the Risk of Preterm Delivery: A Large Scale Cohort Study, Glob J Health Sci, 2015 Sep, Vol no: 7, ISSN No: 5, Page no: 226-232.

10. Catov J. M, Bodnar L. M, Olsen J, Olsen S, Nohr E. A. Periconceptional multivitamin use and risk of preterm or small-for-gestational-age births in the Danish National Birth Cohort. The American journal of clinical nutrition, 2011, Vol no: 94, ISSN No: 3, Page no:906-912.

11. Dolatian M, Mirabzadeh A, Setareh Forouzan A, Sajjadi H, Alavimajd H, Mahmoodi Z, et al. Relationship between Structural and Intermediary Determinants of Health and Preterm Delivery. Journal of Reproduction \& Infertility, 2014, Vol no: 15, ISSN No: 2, Page no: 78-86. 
12. J. Kaplan and S. Lutsenko, "Copper transport in mammalian cells: special care for a metal with special needs," Journal of Biological Chemistry, vol. 284, ISSN No: 38, Page no: 25461-25465.

13. G. Briggs, R. Freeman, and S. Yaffe, A Reference Guide to Fetal and Neonatal Risk: Drugs in Pregnancy and Lactation, Williams \& Wilkins, Baltimore, Md, USA, 5th edition, 1998, Page no: 121-124.

14. Susan Reinwald Connie M.Weaver, The Health Benefits of Calcium Citrate Malate: A Review of the Supporting Science, Volume 54, 2008, Pages 219-346. 